\title{
ABOUT THE DOUBLE IONIZATION OF AMMONIA AND CARBON DIOXIDE. A COMPARISON BETWEEN PHOTOIONIZATION AND ELECTRON IMPACT
}

\author{
R. LOCHT, M. DAVISTER
}

Département de Chimie Générale et de Chimie Physique, Institut de Chimie, Université de Liège, Bâtiment B6, Sart-Tilman par B-4000 Liège 1, Belgium

W. DENZER, H.W. JOCHIMS and H. BAUMGÄRTEL

Institut fiir Physikalische und Theoretische Chemie, Freie Universität Berlin,

\begin{abstract}
The double ionization of $\mathrm{NH}_{3}$ and $\mathrm{CO}_{2}$ is investigated by photoionization in the 30-60 eV photon energy range and electron impact from 30 to $100 \mathrm{eV}$. The lowest onset for $\mathrm{NH}_{3}{ }^{2+}$ formation, measured for the

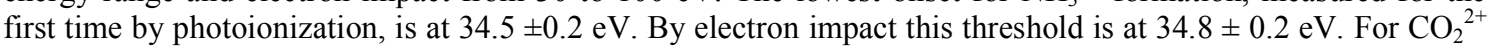
this onset is measured at $37.5 \pm 0.1 \mathrm{eV}$ by photoionization and $38.0 \pm 0.1 \mathrm{eV}$ by electron impact. Close to these onsets two double-ionization energies are observed: $38.5 \pm 0.1 \mathrm{eV}$ by photoionization and $39.5 \pm 0.2 \mathrm{eV}$ by electron impact. These have probably to be ascribed to two different $\mathrm{CO}_{2}{ }^{2+}$ states. Higher-lying doubly ionized states of $\mathrm{CO}_{2}$ are detected in the $60-100 \mathrm{eV}$ electron impact energy range.
\end{abstract}

\section{Introduction}

Compared to the literature devoted to the ionization and dissociation of molecular cations, the abundance of work dedicated to the double ionization of atoms and molecules is very scarce. This statement has to be ascribed to the generally low cross section for double ionization. Until recently, only electron impact, highenergy ion impact and Auger electron spectroscopy allowed us to study these species. The improvement of highenergy light sources, e.g. He(II) resonance lamps and even more the increasing number of electron synchrotron radiation facilities, made it possible to reach photon energies far above the $21.22 \mathrm{eV}$ energy limit. These developments were the starting point of an increasing number of publications in the field of double and even multiple ionization.

The double ionization of $\mathrm{NH}_{3}$ has been investigated by electron impact, from the onset up to $100 \mathrm{eV}$ [1]. The dissociations of $\mathrm{NH}_{3}{ }^{2+}$ into (i) doubly ionized species [1] and (ii) pairs of singly ionized ions were examined by the same method [2-4]. The translational energy distributions of the fragments were measured in the same energy range.

In a recent photoionization work, Samson et al. [5] published the double-ionization cross section of $\mathrm{NH}_{3}$ in the 8-34 $\mathrm{nm}$ wavelength region using synchrotron radiation. They were unable to determine the doubleionization energy of $\mathrm{NH}_{3}$.

The double-ionization energy of $\mathrm{CO}_{2}$ has essentially been investigated by electron impact [6] and Auger electron spectroscopy [7]. The photoionization cross section for $\mathrm{CO}_{2}{ }^{2+}$ has been measured by Samson et al. [8] and the lowest double-ionization energy of $\mathrm{CO}_{2}$ has been determined. More recently the doubly ionized states of carbon dioxide have been studied by charge stripping reaction (CSR) [9] and double-charge transfer spectroscopy (DCTS) [10].

The aim of this investigation is to examine the double-ionization efficiency of $\mathrm{NH}_{3}$ by photoionization from the onset up to $20 \mathrm{~nm}(60 \mathrm{eV})$ and to determine a photoionization onset energy. A direct comparison would be possible with our previous electron impact results [1].

In the frame of a detailed dissociative electronization study of $\mathrm{CO}_{2}$, still in progress [11], the double ionization of $\mathrm{CO}_{2}$ has been investigated by electron impact in the $30-100 \mathrm{eV}$ energy range. For this molecule, the double-ionization efficiency, as observed by photoionization, will also be discussed and compared to 
electroionization. The photoionization efficiencies of the $\mathrm{CO}_{2}{ }^{2+}, \mathrm{CO}^{+}, \mathrm{O}^{+}$and $\mathrm{C}^{+}$are investigated in the same photon energy range.

\section{Experimental}

The electroionization experimental setup used in the present work has been described in detail elsewhere [12,13]. The experimental conditions used for the study of $\mathrm{NH}_{3}$ were extensively set out in a previous publication [1]. The first derivative of the double-ionization efficiency curve is measured and can therefore be directly compared to the photoionization efficiency curves. The critical energies, i.e. the double-ionization energies, are determined by the linear extrapolation method [12].

The $\mathrm{CO}_{2}$ gas sample, purchased from Messer Griesheim and of $99.98 \%$ purity, is used without further purification. For $\mathrm{CO}_{2}^{+}$, the electron energy scale is calibrated by using the appearance energy of $\mathrm{O}^{+} / \mathrm{CO}_{2}$ measured at $19.39 \pm 0.07 \mathrm{eV}$ [11]. As for $\mathrm{NH}_{3}{ }^{2+}$ the first differentiated ionization efficiency curve of $\mathrm{CO}_{2}{ }^{2+}$ has been scanned 300-400 times. This operation has been repeated twelve times. The onset energies reported here are averaged values from these twelve independent measurements. The mentioned error limits are one standard deviation.

In the mass spectrometric photoionization work, the experimental setup used in the abovementioned electron impact experiment has been duplicated. Only the interaction chamber has been modified for photoionization and for fitting different monochromators. The synchrotron radiation of the Berlin electron synchrotron storage ring BESSY has been used as a light source. A TGM monochromator, equipped with a 950 lines $/ \mathrm{mm}$ grating, dispersed the light over the 20-50 nm wavelength region. An aluminum foil is used to filter higher order contributions between $17-34 \mathrm{~nm}$ and the Al-K edge at $16.908 \mathrm{~nm}$ is used to calibrate the wavelength scale. The resolution is $0.1 \mathrm{~nm}$ (i.e. about $0.4 \mathrm{eV}$ at $17 \mathrm{~nm}$ and $0.1 \mathrm{eV}$ at $34 \mathrm{~nm}$ ) over the covered wavelength region. The photoion current is normalized to the photon intensity measured by a venetian-blind photomultiplier with a sodium salicylate covered window.

\section{Results and discussion}

The photoionization efficiency for $\mathrm{NH}_{3}{ }^{2+}$ production in the photon energy range of $30-60 \mathrm{eV}$ is displayed in fig. 1. Notwithstanding the low intensity of the ion signal at $m / e=8 . .5$, the fairly steep rise at threshold allowed us to measure an onset fairly easily.

The earlier published ionization efficiency of $\mathrm{NH}_{3}{ }^{2+}$ by electron impact [1] has been inserted in fig. 1 for comparison. Very good agreement is found between both curves. On the other hand, at the present time, this comparison can be extended up to $100 \mathrm{eV}$ photons. Recently Samson et al. [5] have measured the absolute photoionization cross section for $\mathrm{NH}_{3}{ }^{2+}$ formation in the $36-150 \mathrm{eV}$ energy range. When normalizing the electroionization efficiency curve and the photoionization cross section at $47 \mathrm{eV}$, fig. 2 is obtained. Error bars take into account the $5 \%$ error mentioned by the authors [5]. If the general shape is well accounted for by the first derivative of the electroionization efficiency curve, the photoionization cross section is systematically higher by about $10-15 \%$.

The lowest double-ionization energy of $\mathrm{NH}_{3}$, as measured by photoionization and using the linear extrapolation method [12], is found to be $34.5 \pm 0.2 \mathrm{eV}$. Applying the same method to the first differentiated ionization efficiency from electron impact, the first double-ionization energy of $\mathrm{NH}_{3}$ was measured at $34.8 \pm 0.2$ $\mathrm{eV}$ [1]. Higher ionization energies have been reported earlier [1,14] but owing to the low signal-to-noise ratio, no onsets could be measured above $40 \mathrm{eV}$ photon energy.

Being the first direct measurement of the photoionization threshold of $\mathrm{NH}_{3}{ }^{2+}$ formation, the present result could only be compared to photoion-photoion coincidence work [14] where a dissociative $\mathrm{NH}_{3}{ }^{2+}$ state is detected up from $35.4 \pm 0.5 \mathrm{eV}$. By double charge transfer spectroscopy the first double-ionization energy is located at $35.3 \pm 0.7 \mathrm{eV}$ [15]. The assignment of this double ionization energy as well as the dissociative ionization observed at this energy have been discussed in detail previously [1-4].

The photoion yield and the first derivative of the electron impact ionization efficiency curve of $\mathrm{CO}_{2}{ }^{2+}$ in the 30-60 eV energy range are shown in fig. 3. Both curves are comparable between 30 and $50 \mathrm{eV}$. Above $50 \mathrm{eV}$ and up to $60 \mathrm{eV}$, obviously the photoion yield curve reaches a maximum, whereas the electroionization efficiency steadily increases as shown in fig. 4. This figure displays the ionization efficiency measured between 30 and $100 \mathrm{eV}$. 
Hitchcock et al. [16] derived the oscillator strength for $\mathrm{CO}_{2}{ }^{2+}$ formation from electron energy loss and electron-ion coincidence measurements. Their data are included in fig. 4 and have not been normalized to the present data for sake of clarity. Above $53 \mathrm{eV}$ an increase of the oscillator strength has to be noticed and several maxima seem to be present in the $60-80 \mathrm{eV}$ energy range. Samson et al. [8] measured the ratio of $\mathrm{CO}_{2}{ }^{2+} / \mathrm{CO}^{2}$ as a function of the incident photon energy. This ratio is constant from 60 to $100 \mathrm{eV}$.

Fig. 1. First differentiated electron impact (EI) and photoionization (PI) efficiency curves of $\mathrm{NH}_{3}{ }^{2+}$ in the 30-60 $\mathrm{eV}$ energy range. Vertical bars indicate threshold energies; dashed lines indicate the PIPICO onset at $44.5 \mathrm{eV}$ [14].

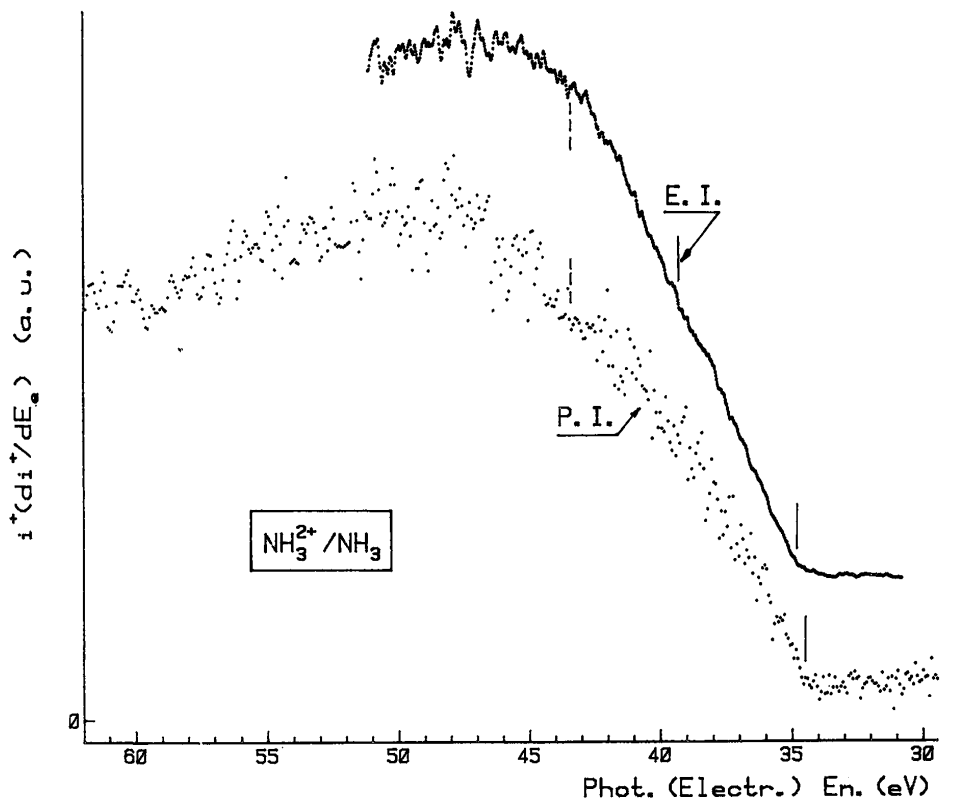

A number of double-ionization energies of $\mathrm{CO}_{2}$ have been determined (see vertical bars in figs. 3 and 4) and are listed in table 1. These energies, averaged over twelve independent measurements, are compared to previous determinations.

The lowest double-ionization energy of $\mathrm{CO}_{2}$ is measured at $38.0 \pm 0.1 \mathrm{eV}$ by electron impact, whereas it is at $37.5 \pm 0.1 \mathrm{eV}$ by photoionization. In both cases the same method is used to determine the threshold energy (linear extrapolation). Notwithstanding the slight discrepancy, both values markedly disagree with the earlier electron impact onset of $36.4 \pm 0.3 \mathrm{eV}$ [6] and the direct photoionization threshold of $36.2 \mathrm{eV}$ [8]. Better agreement is found with the photoionization value of $37.7 \pm 0.3 \mathrm{eV}$ [17] and the Auger electron spectroscopic values of 37.8 and $37.4 \mathrm{eV}$ obtained from the carbon and oxygen K, LL Auger spectra respectively [ 7 ].

Several quantum mechanical calculations were reported to interpret photoionization and Auger electron spectroscopic data on $\mathrm{CO}_{2}$ [17-19]. Keeping in mind the electronic structure of $\mathrm{CO}_{2}$ [18], i.e.

\section{$(\mathrm{O} 1 \mathrm{~s}) 1 \sigma_{\mathrm{g}}^{2}, \quad(\mathrm{O} 1 \mathrm{~s}) 1 \sigma_{\mathrm{u}}^{2}, \quad(\mathrm{C} 1 \mathrm{~s}) 2 \sigma_{\mathrm{g}}^{2}$,}

$$
3 \sigma_{\mathrm{g}}^{2}, 2 \sigma_{\mathrm{u}}^{2}, 4 \sigma_{\mathrm{g}}^{2}, 3 \sigma_{\mathrm{u}}^{2}, 1 \pi_{\mathrm{u}}^{4}, 1 \pi_{\mathrm{g}}^{4} \text {, }
$$

all agree to assign the two hole configuration $\pi_{\mathrm{g}}{ }^{-2}$ to the lowest $\mathrm{CO}_{2}{ }^{2+}$ state. This configuration gives rise to ${ }^{3} \Sigma^{-}$, ${ }^{1} \Sigma^{+}$and ${ }^{1} \Delta$ states [20]. The lowest energy state would correspond to the ${ }^{3} \Sigma_{\mathrm{g}}{ }^{-}$state of $\mathrm{CO}_{2}{ }^{2+}[17]$.

At $1.6 \pm 0.3 \mathrm{eV}$ (by electron impact) or $1.0 \pm 0.2 \mathrm{eV}$ (by photoionization) above the lowest doubleionization energy of $\mathrm{C}_{2}$, i.e. at $39.6 \pm 0.2 \mathrm{eV}$ and $38.5 \pm 0.1 \mathrm{eV}$ respectively, a second onset is detected. Noteworthy is the unusually large discrepancy between the two onsets.

From theoretical calculations an energy separation of $1.4 \mathrm{eV}$ [19] and $1.2 \mathrm{eV}$ [17] is found between the ${ }^{3}$ $\Sigma_{\mathrm{g}}{ }^{-}$and the ${ }^{1} \Delta_{\mathrm{g}}$ states. The ${ }^{1} \Sigma_{\mathrm{g}}{ }^{+}$state is calculated at $1.8 \mathrm{eV}$ above the $\mathrm{CO}_{2}{ }^{2+}$ ground state [17]. The electron 
impact result better fits the $1.8 \mathrm{eV}$ energy interval whereas the $1.2 \mathrm{eV}$ corresponds to the energy separation obtained by photoionization. Though in the averaged electron impact curve a "break" is observed at $38 \mathrm{eV}$, this feature being not easily detected in the individual curves (e.g. obscured by the noise level) is not mentioned in table 1. Likely, owing to lack of resolution and/or a too low signal-to-noise ratio, the ${ }^{1} \Delta_{\mathrm{g}}$ and the ${ }^{1} \Sigma_{\mathrm{g}}{ }^{+}$are not detected by electron impact or photoionization. Consequently it is verylikely that both thresholds are not correlated. The electron impact onset at $39.6 \pm 0.2 \mathrm{eV}$ has to be ascribed to the ${ }^{1} \Sigma_{\mathrm{g}}{ }^{+}$whereas the ${ }^{1} \Delta_{\mathrm{g}}$ state should be assigned to the photoionization threshold at $38.0 \pm 0.1 \mathrm{eV}$.

The photoionization efficiency curves of $\mathrm{CO}_{2}{ }^{+}, \mathrm{CO}^{+}, \mathrm{O}^{+}$and $\mathrm{C}^{+}$have been recorded in the $30-60 \mathrm{eV}$ photon energy range and are displayed in fig. 5. Noteworthy is the observation of a maximum near about $37 \mathrm{eV}$ in all four ion yield curves. A dashed line is drawn at 37.5 and $38.5 \mathrm{eV}$ to tentatively show a correlation between the two first double-ionization energies of $\mathrm{CO}_{2}$ and structures in the decrease of the $\mathrm{CO}_{2}^{+}$photoion yield. For the fragment ions this decrease is smoother.

Fig. 2. First differentiated electron impact ionization efficiency curve of $\mathrm{NH}_{3}{ }^{2+}$ measured between 28 and $100 \mathrm{eV}$ [1] compared to the absolute cross section measurements of Samson et al. [5] (• ). The error is indicated by vertical bars.

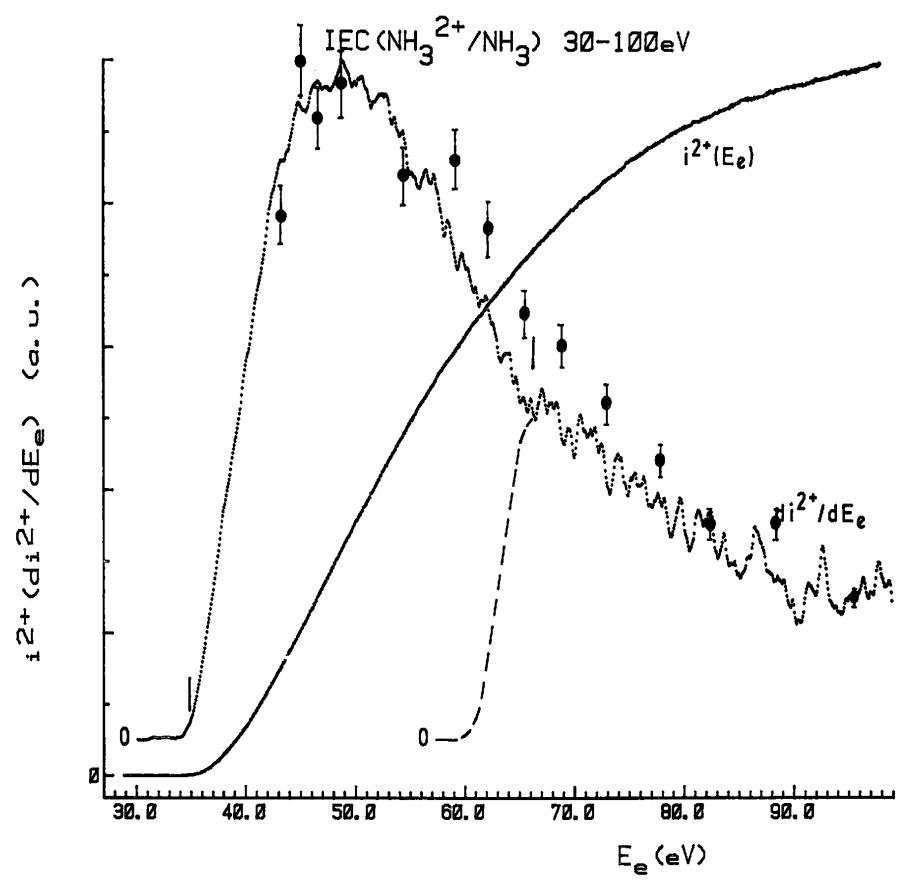

Between 40 and $50 \mathrm{eV}$ electron energy two onsets are measured. The average energies are shown in fig. 3 by vertical bars at $42.9 \pm 0.5$ and $45.9 \pm 0.6 \mathrm{eV}$. By photoionization, besides the onset at $42.3 \pm 0.5 \mathrm{eV}$ a steplike feature has to be mentioned at $41.1 \pm 0.1 \mathrm{eV}$ (dashed line in fig. 3).

To help deciding whether this latter feature has to be ascribed to noise or not, the photoion yield curve of fig. 3 has been Fourier transformed and filtered using different functions and a wide range of band-passes [21]. These tests show that the step-like feature's aspect is largely independent of the band-pass used. To account for this structure a non-resonant autoionization process such as

$$
\begin{aligned}
& \mathrm{CO}_{2}+h \nu \rightarrow \mathrm{CO}_{2}^{+*}+\mathrm{e}^{-} \\
& \stackrel{\longrightarrow}{\longrightarrow} \mathrm{CO}_{2}^{2+}+\mathrm{e}^{-}
\end{aligned}
$$

has to be invoked. In the $\mathrm{CO}_{2}^{+}$photoion yield, singularities are observed in the same energy range as shown in fig. 5 . 
Fig. 3. First differentiated electron impact (EI) and photoionization (PI) efficiency curves of $\mathrm{CO}_{2}{ }^{2+}$ in the 32-62 eV energy range. Vertical bars indicate threshold energies.

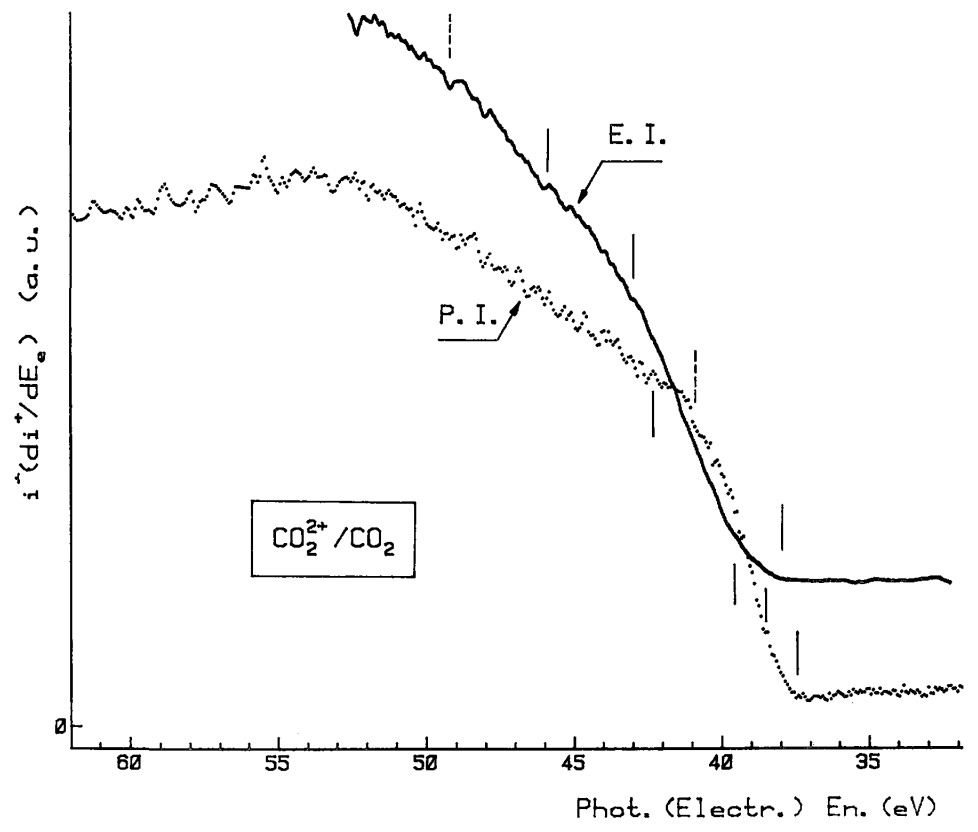

Fig. 4. First differentiated electron impact ionization efficiency curve of $\mathrm{CO}_{2}{ }^{2+}$ measured between 30 and 100 $\mathrm{eV}$. Vertical bars locate critical energies, shaded areas indicate error limits. Comparison with oscillator strength data (•) of Hitchcock et al. [16] drawn on an arbitrary intensity scale.

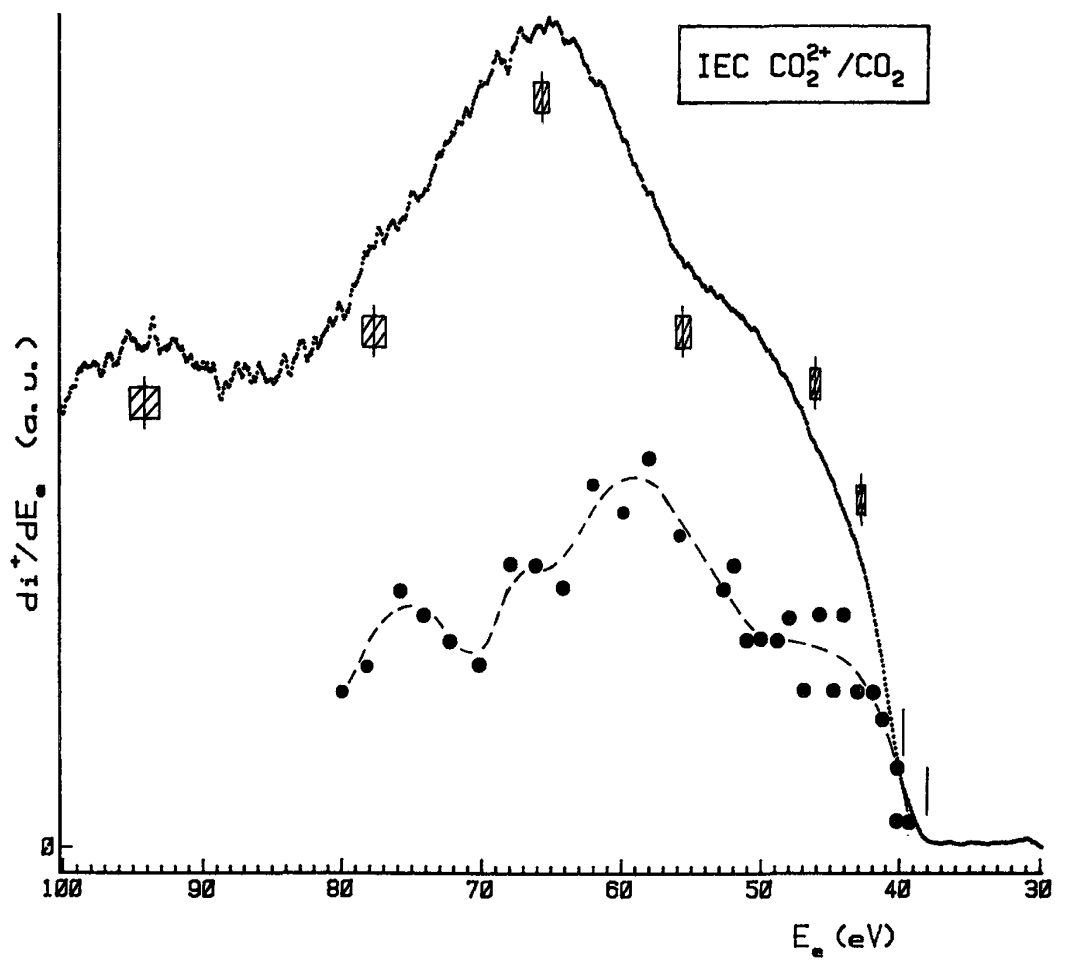


Fig. 5. Photoionization efficiency curves of $\mathrm{CO}_{2}{ }^{2+}, \mathrm{CO}_{2}+, \mathrm{CO}^{+}, \mathrm{O}^{+}$and $\mathrm{C}^{+}$measured in the 30-60 eV photon energy range. "Slope changes" are tentatively correlated (see text).

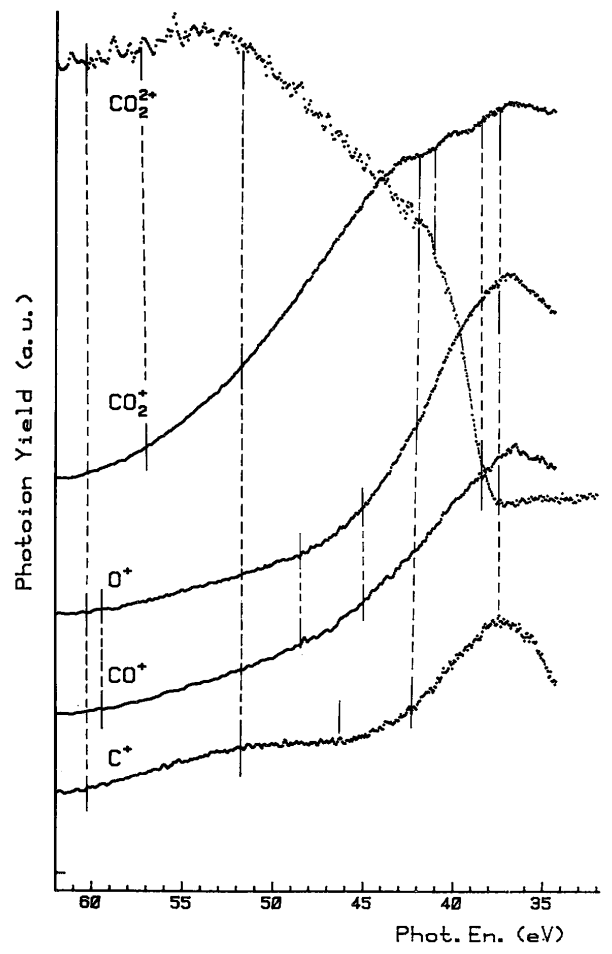

Table 1. Onset energies (eV) determined in the electron impact (EI) and photoionization (PI) efficiency curves of $\mathrm{CO}_{2}{ }^{2+}$. Comparison is made with previous measurements

\begin{tabular}{|c|c|c|c|c|c|c|c|}
\hline \multicolumn{4}{|c|}{ Electron impact } & \multicolumn{4}{|c|}{ Photoionization } \\
\hline this work & ref. [6] & ref. $[10]$ & ref. [16] & this work & ref. [8] & ref. [7] & ref. [17] \\
\hline $38.0 \pm 0.1$ & $36.4 \pm 0.3$ & $38.9 \pm 0.2$ & $\approx 39$ & $37.5 .5 \pm 0.1$ & 36.2 & $37.8,37.4$ & 37.7 \\
\hline $39.6 \pm 0.2$ & - & $40.6 \pm 0.3$ & & $38.5 \pm 0.1$ & & & \\
\hline $42.9 \pm 0.5$ & 41.9 & $42.7 \pm 0.3$ & & $(41.1 \pm 0.1)$ & & & \\
\hline- & & $43.8 \pm 0.3$ & & $42.3 \pm 0.5$ & & & \\
\hline $45.9 \pm 0.6$ & & $45.2 \pm 0.3$ & & & & & \\
\hline- & & $47.4 \pm 0.3$ & & & & & \\
\hline- & & $51.5 \pm 1.0$ & & & & & \\
\hline $55.2 \pm 0.5$ & & $54.0 \pm 1.0$ & & & & & \\
\hline $65.7 \pm 0.6^{\text {a) }}$ & & & $\approx 60, \approx 67^{\mathrm{a})}$ & & & & \\
\hline$\approx 77^{\mathrm{a}}>$ & & & $\approx 76^{\mathrm{a})}$ & & & & \\
\hline $94.5 \pm 1.5^{a)}$ & & & & & & & \\
\hline
\end{tabular}

a) Defined by the maximum.

Above $42 \mathrm{eV}$ a double-ionization energy is determined at $42.9 \pm 0.5 \mathrm{eV}$ (by electron impact) or $42.3 \pm$ $0.5 \mathrm{eV}$ (by photoionization), in good agreement with DCT spectroscopy [10] (see table 1 ) and Auger electron spectroscopy [7].

Mainly two configurations account for this energy [17,18]: $\left(1 \pi_{\mathrm{u}}^{-1}, 1 \pi_{\mathrm{g}}^{-1}\right)$ and $\left(3 \sigma_{\mathrm{u}}^{-1}, 1 \pi_{\mathrm{g}}^{-1}\right) /\left(4 \sigma_{\mathrm{g}}{ }^{-1}, 1 \pi_{\mathrm{g}}{ }^{-1}\right)$ and give rise to singlet or triplet $\Sigma^{+}, \Sigma^{-}$and $\Delta$ and to singlet and triplet $\Pi$ states respectively [20]. By theoretical calculations $[17,18]$, the $\mathrm{CO}_{2}{ }^{2+}$ electronic state at $4.9 \mathrm{eV}$ above the ground state is described as a ${ }^{1} \Pi$.

Whereas above $42 \mathrm{eV}$ the $\mathrm{CO}_{2}{ }^{2+} "$ photoion intensity increases almost linearly, the electron impact 
ionization efficiency shows at least one threshold at $45.9 \pm 0.6 \mathrm{eV}$. The onset marked by a dashed line in fig. 3 at about $49 \mathrm{eV}$ only shows up in the averaged ionization efficiency curve.

By Auger electron spectroscopy [7] a double-ionization energy is observed at $46.2 \pm 0.3$ and at $45.2 \pm$ $0.3 \mathrm{eV}$ by DCT spectroscopy [10]. In the 7.8-8.6 eV energy range above the $\mathrm{CO}_{2}{ }^{2+}$ ground state, theoretical calculations agree to predict the existence of three singlet states [18] and one triplet state [17] arising from $\left(1 \pi_{\mathrm{u}}^{-1}\right.$, $\left.1 \pi_{\mathrm{g}}^{-1}\right), 3 \sigma_{\mathrm{u}}^{-2}$ and $1 \pi_{\mathrm{u}}^{-2}$ configurations. Both ${ }^{1} \Delta_{\mathrm{g}}$ [18] or ${ }^{1} \Sigma^{+}$[17] symmetries have been assigned to this $\mathrm{CO}_{2}{ }^{2+}$ state.

A shown in fig. 5 , in the $42-52 \mathrm{eV}$ photon energy range (marked by dashed lines) where the $\mathrm{CO}_{2}{ }^{2+}$ ion yield increases linearly, the intensity of $\mathrm{CO}_{2}{ }^{+}$decreases almost linearly. Up from $52 \mathrm{eV}$ the $\mathrm{CO}_{2}{ }^{2+}$ ion yield reaches a maximum at about $54 \mathrm{eV}$ and slowly decreases between 56 and $62 \mathrm{eV}$. In the same energy ranges, if the $\mathrm{CO}_{2}{ }^{+}$ion intensity continuously decreases, slope changes have to be noticed at about 52 and $60 \mathrm{eV}$.

Simultaneously (see fig. 5) for the fragment ions $\mathrm{O}^{+}, \mathrm{CO}^{+}$and $\mathrm{C}^{+}$the intensity decrease occurs less regularly. For the latter species the ion yield is even constant in the 46-52 eV photon energy range. On the other hand, at about 45, 48.5 and $59 \mathrm{eV}$ "breaks" are observed in $\mathrm{CO}^{+}$and $\mathrm{O}^{+}$ionization efficiency curves. Very likely this would be indicative of $\mathrm{CO}^{+}+\mathrm{O}^{+}$pair production at the expense of doubly ionized $\mathrm{CO}_{2}$ states. An analogous correlation could be made for the $\mathrm{C}^{+}+\mathrm{O}^{+}$pair at 42 and $60 \mathrm{eV}$.

In the 50-100 eV energy range, the electron impact ionization efficiency curve shows an onset at $55.2 \pm$ $0.5 \mathrm{eV}$ and maxima at $65.7 \pm 0.7 \mathrm{eV}$ and $94.5 \pm 1.5 \mathrm{eV}$ and a shoulder at about $77 \mathrm{eV}$. As shown in fig. 4, by the comparison with the data published by Hitchcock et al. [16], the broad maximum at $65.7 \mathrm{eV}$ probably includes several processes. This is very likely the case at $94.5 \mathrm{eV}$ too. Auger transitions were observed up to $55 \mathrm{eV}$ above the $\mathrm{CO}_{2}{ }^{2+}$ ground state and theoretical calculations have been performed up to the same energy [18].

At about $17.2 \pm 0.7 \mathrm{eV}$ above the $\mathrm{CO}_{2}{ }^{2+}$ ground state fairly well isolated states are predicted [18], corresponding to ${ }^{1} \Sigma_{\mathrm{u}}\left(4 \sigma_{\mathrm{g}}{ }^{-1}, 3 \sigma_{\mathrm{u}}{ }^{-1}\right)$ and ${ }^{1} \Sigma_{\mathrm{g}}\left(4 \sigma_{\mathrm{g}}{ }^{-2}\right)$ and lying at 16.3 and $18.1 \mathrm{eV}$ above the lowest doubly ionized state. Either or both states can account for the onset observed at $55.2 \pm 0.5 \mathrm{eV}$.

To the broad maximum at $65.7 \mathrm{eV}$, i.e. about $28 \mathrm{eV}$ above the $\mathrm{CO}_{2}{ }^{2+}$ ground state, could correspond the Auger transitions observed at $60.2,61.1$ and $64.8 \mathrm{eV}$ [7]. The shoulder at $77 \mathrm{eV}$, corresponding to the maximum at $76 \mathrm{eV}$ by Hitchcock et al. [16], could be correlated with the Auger transition observed in the oxygen K, LL spectrum at $76.8 \mathrm{eV}$. This transition is assigned to a ${ }^{1} \Sigma_{\mathrm{g}}\left(3 \sigma_{\mathrm{g}}{ }^{-1}, 4 \sigma_{\mathrm{g}}^{-1}\right)$ state [17]. In the same Auger spectrum a transition is finally observed at $94.8 \mathrm{eV}$ [7] which could be related to the peak observed at $94.5 \mathrm{eV}$ in the present experiment. However, a contribution from the $89 \mathrm{eV}$ transition [7] to this peak has also to be considered.

\section{Acknowledgement}

We acknowledge the Fonds de la Recherche Fondamentale Collective (FRFC) and the University of Liège for financial support and the Belgian Government for an ARC contract. This work is part of the collaboration program of the Département de Chimie, University of Liège, and the Institut fur Physikalische Chemie, Freie Universität, Berlin. This collaboration was made possible by financial support of the Fonds National de la Recherche Scientifique (FNRS ) and the Bundesministerium fur Forschung und Technologie. Both are gratefully acknowledged.

\section{References}

[1] R. Locht and J. Momigny, Chem. Phys. Letters 138 ( 1987 ) 391.

[2] R. Locht, Ch. Servais, M. Ligot, Fr. Derwa and J. Momigny, Chem. Phys. 123 (1988) 443.

[3] R. Locht, Ch. Servais, M. Ligot, M. Davister and J. Momigny, Chem. Phys. 125 (1988) 425.

[4] R. Locht and J. Momigny, Chem. Phys. 127 ( 1988) 435.

[5] J.A.R. Samson, G.N. Haddad and L.D. Kilcoyne, J. Phys. Chem. 87 (1987) 6416.

[6 ] F.H. Dorman and J.D. Morrison, J. Chem. Phys. 35 (1961) 575. 
Published in: Chemical Physics (1989), vol. 138, pp. 433-440.

Status: Postprint (Author's Version)

[7] W.E. Moddeman, T.A. Carlson, M.O. Krauss, B.P. Pullen, W.E. Bull and G.K. Schweitzer, J. Chem. Phys. 55 (1971) 2317.

[8] J.A.R. Samson, P.C. Kemeny and G.N. Haddad, Chem. Phys. Letters 51 (1977) 75.

[9] C.J. Porter, C.J. Proctor, T. Ast and J.H. Beynon, Croat. Chem. Acta 54 (1981) 407.

[10] P.G. Fournier, J. Fournier and M. Mousselmal, to bepublished.

[11] M. Davister and R. Locht, to be published.

[12] R. Locht and J. Schopman, Intern. J. Mass Spectrom. Ion Phys. 15 (1974) 361.

[13] Ch. Servais, R. Locht and J. Momigny, Intern. J. Mass Spectrom. Ion Phys. 74 (1986) 179.

[14] D. Winkoun and G. Dujardin, Z. Physik D 4 (1986) 57.

[15 ] J. Appel and J.A. Horsley, J. Chem. Phys. 60 (1974) 3445.

[16] A.P. Hitchcock, C.E. Brion and M.J. van der Wiel, Chem. Phys. 45 (1980) 461.

[17] P. Millie, I. Nenner, P. Archirel, P. Lablanquie, P. Fournier and J.H.D. Eland, J. Chem. Phys. 84 (1986) 1259.

[18 ] H. Ågren, J. Chem. Phys. 75 (1981) 1267.

[19] J.A. Kelber, D.R. Jennison and R.R. Rye, J. Chem. Phys.75 (1981) 652.

[20] G. Herzberg, Molecular Spectra and Molecular Structure, Vol. 2. Infrared and Raman spectra of polyatomic molecules; Vol. 3. Electronic spectra and structure of polyatomic molecules (Van Nostrand, Princeton, 1967).

[21] Ch. Servais, Mémoire de licence, Université de Liège,Belgium (1983). 